\title{
Sensitive HPV Genotyping Based on the Flow-Through Hybridization and Gene Chip
}

\author{
Pingping Tao, ${ }^{1}$ Weiping Zheng, ${ }^{1}$ Yungen Wang, ${ }^{1}$ and Mei-lu Bian ${ }^{2}$ \\ ${ }^{1}$ Department of Gynecology, Shaoxing People's Hospital and Shaoxing Hospital of Zhejiang University, Shaoxing 312000, China \\ ${ }^{2}$ Department of Obstetrics and Gynecology, China-Japan Friendship Hospital, No. 2 Yinghua Dongjie, Hepingli, \\ Beijing 100029, China
}

Correspondence should be addressed to Mei-lu Bian, bianmeilu@yahoo.cn

Received 13 July 2012; Revised 7 September 2012; Accepted 13 September 2012

Academic Editor: F. C. Schmitt

Copyright (c) 2012 Pingping Tao et al. This is an open access article distributed under the Creative Commons Attribution License, which permits unrestricted use, distribution, and reproduction in any medium, provided the original work is properly cited.

\begin{abstract}
Persistent infection of high-risk human papillomavirus (HPV) has been recognized as the direct cause of cervical carcinoma. Therefore, detection and genotyping of HPV are important to cervical-cancer screening. In this study, we have evaluated the efficacy of flow-through hybridization and gene chip (HybriMax) on HPV genotyping through comparison of the results with Hybrid Capture II (HC-II) and in situ hybridization (ISH). 591 women were classified into 6 groups according to their histological diagnoses. The overall accordance rate on 13 types of HPV genotypes between HybriMax and HC-II were 92.5\% and 100\% in the cancer group. The overall accordance was excellent with the Kappa index (KI) of 0.814 . The value of KI in each group was 0.750 (normal cytological diagnosis), 0.781 (chronic cervicitis), 0.80 (condyloma acuminatum), 0.755 (cervical intraepithelial neoplasia (CIN) I), 0.723 (CIN II), and 0.547 (CIN III) $(0.75>\mathrm{KI}>0.4$, good; KI $\geq 0.75$, excellent). The 10 most common HPV subtype detected by HybriMax were 16, 52/58, 18, 33, 31, 81, 53, 68, and 66 in patients, and 16, 68, 18, 52, 58, 11, 53, 31/39, and 33 in normal controls. In conclusion, HybriMax is an efficient method for HPV genotyping and more suitable for clinical use.
\end{abstract}

\section{Introduction}

The human papillomavirus (HPV) belongs to the Papillomaviridae family and persistent infection of high-risk HPV is the direct cause of cervical carcinoma, which is the second most common malignancy among women worldwide [1]. HPV detection and genotyping is the most effective and accurate approach in screening of the early cervical lesions and cervical cancer. With HPV genotyping becoming more prevalent, over 120 types of HPV have been identified, of which at least 40 types are indicated to infect the genital epithelium [2]. Its genotypes are generally classified into high-risk (HR-) and low-risk (LR-) groups based on their carcinogenic potential. HR-HPVs include HPV 16, 18, 31, $33,35,39,45,51,52,56,58,59,68,73,82$, and so on, and the LR-HPVs include 6, 11, 40, 42, 43, 44, 54, 61, 70, 72,81, cp6108, and so on [3]. It should be noted that it is of particular interest to know the difference in the clinical properties of cervical neoplasia according to HPV types, which will help us in estimating the gravity of the disease and evaluating the prognosis after therapy according to different HPV types. Although there are geographical differences in the distribution of HPV types among populations, globally, it has been shown that HPV 16, 18, 45, 33, and 31 are the most prevalent HPV types associated with cervical cancer [3]. Effective HPV vaccines might be a new prophylaxis for cervical diseases. It is of indicated that a vaccine that included the 7 most common HPV types can prevent $87.4 \%$ of cervical cancer worldwide [4].

Therefore, it is of considerable clinical value to establish a reliable and convenient method to detect and genotype HPV [5]. Currently, being the only FDA-approved (the U.S. Food and Drug Administration) commercially available method for the detection of HPV DNA, the well-established Hybrid Capture II system (HC-II) has been proven to be a sensitive and reliable assay, which can detect 13 types of carcinogenicHPV types or 5 types of low-risk HPV in a single test $[6,7]$. However, its main undeniable limitation is that HC-II cannot 
distinguish between different HPV genotypes definitely [5, 8]. Recently, a new HPV-genotyping method combining two advanced techniques, the flow-through hybridization and gene chip (HybriMax) has been used to detect and genotype HPV, which can distinguish 21 different types of HPV DNA in a single test and diagnose multiple infections [9-11]. In this study, we have evaluated the efficacy of HybriMax on cervical HPV genotyping through comparison of the results with Hybrid Capture II (HC-II) and in situ hybridization (ISH). We showed that the most common HPV types tested by HybriMax in different grades of cervical disease could be determined, suggesting that HybriMax is an efficient method for HPV genotyping and is more suitable for clinical use.

\section{Material and Methods}

2.1. Study Populations. 591 out of 7520 women who accepted liquid-based cytology examination in China-Japan Friendship Hospital from August 2004 to May 2005 were randomly selected for detection of the $21 \mathrm{HPV}$ genotypes by HybriMax, and their mean age was $35.4 \pm 7.7$ (ranging from 20 to 64). Among them, 138 women (mean age was $35.8 \pm 7.8$ ), who diagnosed within normal limits with cervical cytology for at least two years without any cervical disease or operation, were described as "normal". A total of 453 women were diagnosed with abnormal cytology, and the mean age was 34.1 \pm 6.9. Cytological diagnosis (according to the 2001 Bethesda System) of those 453 patients were as follows: 161 cases with atypical squamous cells (ASC), 187 cases with low-grade squamous intraepithelial lesion (LSIL), 105 cases with high-grade squamous intraepithelial lesion (HSIL), or squamous cell carcinoma (SCC). Patients were classified into 6 groups according to their histopathology diagnosis from specimens of olcposcopic biopsy, loop electrosurgical excision procedure (LEEP), or cold-knife conization. These 6 groups include 152 cases of chronic cervicitis, 101 cases of cervical intraepithelial neoplasia (CIN, which indicates that dysplasia is seen on a biopsy of the cervix) I (mild dysplasia), 77 cases of CIN II (moderate to marked dysplasia), 76 cases of CIN III (severe dysplasia to carcinoma in situ), 27 cases of SCC, and 20 cases of condyloma acuminata.

With liquid-based cytology, samples were taken with the cervical brush at gynecological examination for HPV DNA testing. The collected specimens were stored at $4^{\circ} \mathrm{C}$ and processed within two weeks. It is prohibitive to apply vaginal douching three days prior to the collection of samples or to have sexual intercourse within one day. Sampling should not be carried out during menstrual period. All the positive cytological samples were confirmed by biopsies.

2.2. HPV Genotyping by HybriMax. HPV genotyping by HybriMax was performed using an HPV GenoArray Test Kit (HybriBio Ltd., Chaozhou, China). This assay can determine $21 \mathrm{HPV}$ types, including 14 high-risk HPV types $(16,18,31$, $33,35,39,45,51,52,56,58,59,66$, and 68 ), five low-risk HPV types (6, 11, 42, 43, and 44), and two unknown-risk types (53 and CP8304), by the flow-through hybridization technique using HPV DNA amplified by PCR. In brief,
$0.5 \mathrm{~mL}$ specimen was centrifuged at $13,000 \mathrm{~g}$ for $15 \mathrm{~min}$ with KUBOTA 6930. Then, the supernatant was removed and the pellet was resuspended in $200 \mu \mathrm{L}$ PBS buffer. DNA was extracted with the QIAamp Mini Kit (Qiagen, Germany). High-quality DNA was yielded from lysis of cells by isolation of DNA, precipitation, and purification. The instrument used for PCR amplification was PE 9600 thermal cycler (Applied Biosystems, USA). We prepared the PCR master mix by mixing $19.25 \mu \mathrm{L}$ PCR-mix solution and $0.75 \mu \mathrm{L}$ DNA Taq polymerase for each reaction, adding $5 \mu \mathrm{L}$ DNA template in each tube, and then running the amplification program. A positive control and a negative control were included in each PCR analysis process. The amplification program was denaturing at $95^{\circ} \mathrm{C}$ for $9 \mathrm{~min}, 40$ cycles at $95^{\circ} \mathrm{C}$ for 20 seconds, $55^{\circ} \mathrm{C}$ for 30 seconds, and $72^{\circ} \mathrm{C}$ for 30 seconds, and finally extension at $72^{\circ} \mathrm{C}$ for $5 \mathrm{~min}$. The primer used was MY09/11 primer system.

The flow-through hybridization was made on a prewarmed instrument at $45^{\circ} \mathrm{C}$, and the HybriMem HPV-21 DNA microarray membrane was placed, which is marked with $21 \mathrm{HPV}$-genotype probes including HPV 6, 11, 42, 43, $44,16,18,31,33,35,39,45,51,52,53,56,58,59,66,68$, and cp8304. The number of samples tested in a batch could be adjusted from 1 to 15 as required. The PCR products were denatured at $95^{\circ} \mathrm{C}$ for $5 \mathrm{~min}$ just before hybridization and then was chilled on ice for at least $2 \mathrm{~min}$. We mixed the PCR products with hybridization solutions and then added the mixture into sample wells to proceed with flowthrough hybridization for about 5-10 min. The membrane was washed with hybridization solution, and the empty space was blocked without reaction. Adding NBT/BCIP solution to display the results, a positive result was indicated by a clearly visible indigo dot. The HPV-genotype result was determined according to the position of the HPV-genotype probes on the microarray chip. Multiple dots indicated multiple infections.

2.3. HPV Testing by HC-II. 413 samples were detected by the commercially available HC-II assay (Digene Co., Gaitherburg, MD, USA). The probes used were designed to detect 13 types of high-risk HPV, including 16, 18, 31, 33, $35,39,45,51,52,56,58,59$, and 68 . This enzyme-linked immunosorbent assay is based on a sandwich hybridization followed by a nonradioactive alkaline phosphatase reaction with chemiluminescence in the microplate. Samples were classified as positive for HPV DNA if their chemiluminescence was more than $1.0 \mathrm{pg} / \mathrm{mL}$ of control.

2.4. HPV 16/18 Testing by ISH. 101 paraffin-embedded specimens were detected by ISH for HPV 16/18. It was performed with REMBRANDT universal DISH and AP Detection Kit (PanPath, The Netherlands). After being dewaxed and hydrated, paraffin sections $(5-6 \mu \mathrm{m})$ were incubated in diluted proteolytic solution at $37^{\circ} \mathrm{C}$ for $30 \mathrm{~min}$ and dehydrated. Specimens were denatured at $95^{\circ} \mathrm{C}$ for $5 \mathrm{~min}$ by applying the probe solution to them and hybridized at $37^{\circ} \mathrm{C}$ for 2 hours. Then, they were incubated with Pan Wash at $37^{\circ} \mathrm{C}$ for $15 \mathrm{~min}$ except the positive control. We dropped the conjugate by heating for $30 \mathrm{~min}$ and the NBT/BCIP 
TABLe 1: Correlation between HybriMax and HC-II in detecting HPV DNA.

\begin{tabular}{|c|c|c|c|c|}
\hline \multirow{2}{*}{ Groups } & \multirow{2}{*}{ Cases } & \multicolumn{2}{|c|}{ Positive rate $\%(n)$} & \multirow{2}{*}{ Kappa index } \\
\hline & & HC-II & HybriMax & \\
\hline Normal & 79 & $24.0(19)$ & $31.6(25)$ & 0.75 \\
\hline Chronic cervicities & 110 & $76.3(84)$ & $81.8(90)$ & 0.781 \\
\hline CIN I & 82 & $82.9(68)$ & $80.4(66)$ & 0.755 \\
\hline CIN II & 48 & $87.5(42)$ & $87.5(42)$ & 0.619 \\
\hline CINI III & 56 & $91.1(51)$ & $96.4(54)$ & 0.548 \\
\hline SCC & 18 & $100(18)$ & $100(18)$ & - \\
\hline Condyloma acuminata & 20 & $0.5(10)$ & $0.4(8)$ & 0.8 \\
\hline Total & 413 & $70.7(292)$ & $73.4(303)$ & 0.814 \\
\hline
\end{tabular}

If Kappa index $\geq 0.75$, the accordance rates were regarded as excellent; if $0.4 \leq$ Kappa index $\leq 0.75$, the accordance rates were regarded as good. Abbreviations: CIN I: cervical intraepithelial neoplasia grade I; CIN II: cervical intraepithelial neoplasia grade II; CINI III: cervical intraepithelial neoplasia grade III; SCC: squamous cell carcinoma.

TABLe 2: The accordance of HybriMax and ISH for HPV $16 / 18$ detection.

\begin{tabular}{lccc}
\hline & HybriMax & ISH \\
\hline Positive cases $(n)$ & 63 & & 56 \\
Positive rate (\%) & 62.4 & & 55.4 \\
Accordance rate (\%) & & 89.1 & \\
Kappa index & & 0.776 & \\
\hline
\end{tabular}

substrate by heating for $10 \mathrm{~min}$ at $37^{\circ} \mathrm{C}$ in the dark and finally counterstaining and mounting.

2.5. Statistical Analysis. The statistical analysis was performed using the SPSS software (version 10.0) for chisquare test and Kappa index was calculated to evaluate the accordance of the results.

\section{Results}

3.1. The Correlation of HybriMax and HC-II. The positive rate of HybriMax for 13 types of HR-HPV (detected by HCII) was $73.7 \%(303 / 413)$ and the positive rate of HC-II was $70.7 \%$ (292/413) (Table 1). In general, HPV-detection rates by HybriMax agreed well with those of HC-II with a total accordance rate of $92.5 \%$. It is shown that the accordance rates were excellent in general (Kappa index $(\mathrm{KI})=0.814)$ in the group with normal cytological diagnosis $(\mathrm{KI}=0.750)$, chronic cervicitis $(\mathrm{KI}=0.781)$, condyloma acuminata $(\mathrm{KI}=$ $0.80)$, group CINI $(\mathrm{KI}=0.755)$, CIN II $(\mathrm{KI}=0.723)$, and good at group CIN III $(\mathrm{KI}=0.547)(0.75>\mathrm{KI}>0.4$, good; $\mathrm{KI} \geq 0.75$, excellent).

In addition, we found that there were 21 cases with HCII-negative and HybriMax-positive diagnoses, including 8 cases of HPV18 (or multiple infections that include HPV18), 5 cases of HPV 68 (or that include HPV 68), 4 cases of HPV 31 (or that include HPV 31), 2 cases of HPV 33 (or that include HPV 33), and 2 cases of HPV 51 (or that include HPV 51). Another two cases with genotyping results of HPV 66, 2 cases with HPV 6, one case with HPV 6/11, and one case with HPV 44 by HybriMax were HC-II positive, the results of which may be out of the HC-II testing limits.

3.2. The Correlation of HybriMax and ISH. Table 2 showed the accordance of the HPV 16/18 results of HybriMax and ISH. It is shown that the accordance rate of the two methods was $89.1 \%$, and their Kappa index was 0.776 .

3.3. The Positive Rates of Different Genotypes in Each Group. Table 3 showed the positive rates of different genotypes in each group. There were significant differences between the normal group and abnormal groups. The total HPV-positive rate of patients with abnormal cytological diagnosis was $89.6 \%$ (406/453), with $80.9 \%$ in group A, $90.1 \%$ in group B, $92.2 \%$ in group C, $97.4 \%$ in group D, and $100 \%$ in groups $\mathrm{E}$ and $\mathrm{F}$. In the group of patients with normal cytological diagnosis, the positive rate was $30.4 \%$.

The 10 most common genotypes and their infection rates with abnormal cytological diagnosis in turn (descending) were HPV 16 (28.9\%), 52/58 (19.0\%), 18 (16.8\%), 33 (9.9\%), $31(9.7 \%), 81(8.4 \%), 53(8.6 \%), 68(8.4 \%), 66(5.1 \%)$, and $43(0 \%)$. The 10 most common genotypes in normal groups were HPV 16 (8.0\%), 68 (7.2\%), 18 (6.5\%), 52/58 (3.6\%), 11 (2.9\%), $53(2.2 \%), 31 / 39(1.4 \%)$, and $33(0.7 \%)$, while HPV $35,45,59,66,42,43$, and 44 were not detected.

The 10 most common genotypes in different groups (in descending order) were as follows: HPV 16, 18/58, 52, 31, 53, $68,81,33$, and 39 in the group of chronic cervicitis; HPV $58,16 / 52,18,33,68,53,56 / 81$, and 31 in the group of CIN; HPV 16, 52, 58, 18, 33/81, 31/51, 53, and 68 in CIN; HPV $16,58,52,18,31,33,81,53 / 68$, and 66 in CIN III; HPV $16,18,52,58,33,66,68$, and $31 / 51 / 53$ in group of SCC. We come to the conclusion that HPV 16, 18, 52, 58, 33, and 31 were the 6 most common HPV types that can infect the patients with cervical lesions. The most common HPV types causing condyloma acuminata were HPV 11 (with a total positive rate of $55.0 \%$ ) and HPV 6 (with a total positive rate of $30.0 \%)$.

The positive rates of the 6 most common genotypes in different groups were shown in Figure 1. HPV 16 was the most frequent type in almost all the groups (except 
TABLE 3: Positive rates of different HPV genotypes in each group detected by HybriMax.

\begin{tabular}{|c|c|c|c|c|c|c|c|c|}
\hline \multirow{2}{*}{ HPV type } & \multicolumn{7}{|c|}{ Group no. of patients $\%$} & \multirow{2}{*}{ Normal } \\
\hline & Total & A & B & $\mathrm{C}$ & D & E & $\mathrm{F}$ & \\
\hline 16 & 28.9 & 19.7 & 21.8 & 32.5 & 47.4 & 55.6 & 15.0 & 8.0 \\
\hline 18 & 16.8 & 14.5 & 14.9 & 15.6 & 21.1 & 25.9 & 20.0 & 6.5 \\
\hline 31 & 9.7 & 11.2 & 6.9 & 10.4 & 13.2 & 3.7 & 5.0 & 1.4 \\
\hline 33 & 9.9 & 6.6 & 11.9 & 13.0 & 10.5 & 14.8 & 5.0 & 0.7 \\
\hline 35 & 0.2 & 0.7 & - & - & - & - & - & - \\
\hline 39 & 4.2 & 5.3 & 4.0 & 3.9 & 3.9 & - & 5.0 & 1.4 \\
\hline 45 & 0.6 & 0.7 & 1.0 & 1.3 & - & - & - & - \\
\hline 51 & 4.0 & 3.3 & 2.0 & 10.4 & 2.6 & 3.7 & - & 0.7 \\
\hline 52 & 19.0 & 11.8 & 21.8 & 26.0 & 25.0 & 22.2 & 5.0 & 3.6 \\
\hline 53 & 8.6 & 10.5 & 8.9 & 6.5 & 6.6 & 3.7 & 15.0 & 2.2 \\
\hline 56 & 4.0 & 4.6 & 7.9 & 2.6 & 1.3 & - & - & 0.7 \\
\hline 58 & 19.1 & 14.5 & 22.8 & 18.2 & 28.9 & 18.5 & - & 3.6 \\
\hline 59 & 1.1 & 1.3 & 2.0 & 1.3 & - & - & - & - \\
\hline 66 & 5.1 & 3.9 & 5.9 & 3.9 & 5.3 & 11.1 & 5.0 & - \\
\hline 68 & 8.4 & 9.9 & 10.9 & 6.5 & 6.6 & 7.4 & - & 7.2 \\
\hline 81 & 8.8 & 8.6 & 7.9 & 13.0 & 7.9 & 3.7 & 10.0 & 0.7 \\
\hline 6 & 2.9 & 0.7 & 5.0 & 1.3 & 1.3 & - & 30.0 & 0.7 \\
\hline 11 & 4.4 & 2.6 & 3.0 & - & 2.6 & - & 55.0 & 2.9 \\
\hline 42 & 0.2 & 0.7 & - & - & - & - & - & - \\
\hline 43 & - & - & - & - & - & - & - & - \\
\hline 44 & 0.8 & - & 2.0 & 1.3 & - & - & 5.0 & - \\
\hline
\end{tabular}

Group A: chronic cervicitis; group B: CIN I; group C: CIN II; group D: CIN III; group E: SCC; group F: condyloma acuminata.

for CIN I, less than HPV 58). Furthermore, the positive rate increased with the development of the disease. HPV 18 was the second most frequent type in SCC, but the fourth most frequent type in all groups of CIN. Similarly, the positive rates of HPV 18 increase with the development of disease. HPV 52 and HPV 58 were the third and fourth most common types in the group of SCC, but the second and third most frequent types in all groups of CIN. HPV 33 was the fifth most frequent type in the group of SCC, and the positive rates increase with the progress of disease in general.

\section{Discussion}

Previous studies have documented that HPV plays a central role in the etiology of cervical cancer [12, 13]. It is indicated that women positive for HPV DNA have a risk of developing cervical cancer 15-50 times higher than those without HPV DNA. Therefore, it is a preferred approach to combine the liquid-based cytology diagnosis with HPV DNA testing in cervical-cancer screening [14]. Traditional HPVgenotyping methods, such as Southern-blot hybridization, direct sequencing [15], and restriction fragment length polymorphisms (RFLP) [16] based on polymerase chain reaction (PCR) are unsuitable for clinical use due to various reasons, such as low sensitivity, difficultly of handling, and time consumption. ISH is an easy to handle, reliable method

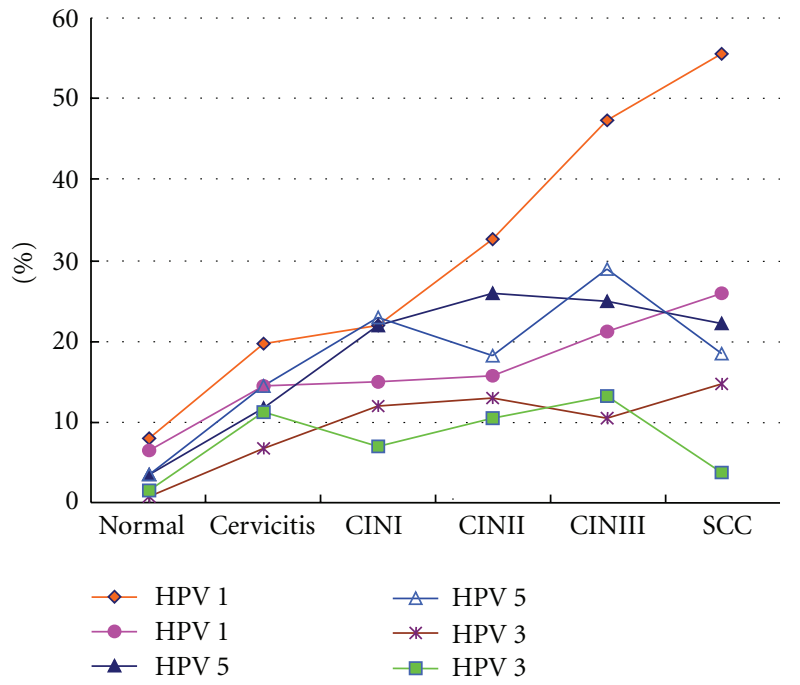

Figure 1: The positive rates of the 6 most common genotypes in different groups. It shows the positive rates of the 6 most common genotypes in different groups. CIN: cervical intraepithelial neoplasia, SCC: squamous cell carcinoma. HPV 16 was the most frequent type in almost all the groups (except for CIN I, less than HPV 58); the positive rate increased with the development of disease. Abbreviations: CIN I: cervical intraepithelial neoplasia grade I; CIN II: cervical intraepithelial neoplasia grade II; CINI III: cervical intraepithelial neoplasia grade III; SCC: squamous cell carcinoma. 
for HPV detection and typing, working on PAP smears and paraffin-embedded sections $[17,18]$; however, its sensitivity and genotype detection are limited. HC-II, as the most wildly accepted HPV-DNA-testing method for clinical use, is considered to be reliable, sensitive and easy to handle $[6,7]$. However it has a limitation in discriminating HPV genotype and multiple infections, for HPV infection can only be attributed to a "low-risk" or "high-risk" group $[5,8]$.

Flow-through hybridization is the most efficient method for molecular hybridization [9-11]. A newly developed biotechnology named HybriMax, combining two advanced techniques, the flow-through hybridization and gene chip, is developeded to be used in clinical practice for the detection and genotyping of 21 different types of HPVs at one test. Multiple infections can be detected at one time. The HPV genotypes detected by HybriMax include not only the 5 lowrisk HPVs and 13 high-risk HPVs that HC-II can detect, but also HPV 66, 53, and cp8304 that HC-II cannot detect. In addition, HybriMax provides much more information than that afforded by HC-II. In this study, our results indicated that HybriMax was highly comparable to HC-II in the detection of 13 types of HR-HPV, and has good accordance with ISH in the detection of HPV 16/18. Therefore, it is suggested that HybriMax can serve as an ideal method for HPV genotyping.

Among the 21 cases which HC-II diagnosed negative while HybriMax revealed positive, there were 8 cases of HPV 18 and 5 cases of HPV 68, which probably suggested that HC-II was less sensitive to those HPV types. There were some samples detected HPV 6, 11, 44, and 66 positive by HybriMax, present also positive by HC-II, which should be negative (not included in the range of genotype which can detected by HC-II). It was indicated that there was crossreaction between the probes of the HC-II HPV types. It had been reported that the probes of HC-II can have a cross-reaction with less than 22 types of HPV DNA other than 13 types of HC-II [19]. However, we do not exclude the possibility of false positive by HybriMax. A potential disadvantage of HybriMax comes from the procedure of PCR, which generally was confronted with the problem of contamination. For this reason we should be more careful to do the procedure.

In addition, this study revealed that, in China, the 6 most common genotypes in cervical lesions were HPV 16, $18,52,58,33$, and 31 included in cervical cancer. The recent international prevalence surveys by the International Agency of Research on Cancer (IARC) reported that the most common HPV types of invasive cancer were $16(57.4 \%)$, $18(16.6 \%), 45(6.8 \%), 31(4.3 \%), 33(3.7 \%), 52(2.5 \%)$, $58(2.3 \%), 35(2.2 \%), 59(1.5 \%)$, and $56(1.3 \%)$, but the study did not include the Chinese population [4]. A metaanalysis made by Clifford in 2003 revealed that in cases from Asia, HPV $58(5.8 \%)$ and $52(4.4 \%)$ were more common than HPV 45, 31, and 33 [20], which supports our results. A large-scale survey on the HPV types of 809 cervical cancer cases in China showed that HPV 16 and HPV 18 were the first and second most common HPV types, and HPV 58 and 52 were the third and fourth most common genotypes, followed by 31 and 33, which also supported our results [21].

This study also found that the positive rates of HRHPVs in groups of abnormal cytological diagnoses were prominently higher than that of normal groups. It has been revealed that abnormality of cytology is strongly related to HPV infection. Therefore, it is suggested that HPV detection is especially important for women with abnormal cytological findings.

\section{Acknowledgment}

This study was supported by Zhejiang provincial key medical discipline construction program of China (no. GJSX-010004).

\section{References}

[1] C. M. McLachlin, "Human papillomavirus in cervical neoplasia: role, risk factors, and implications," Clinics in Laboratory Medicine, vol. 20, no. 2, pp. 257-270, 2000.

[2] M. Poljak, "Review of 20 years of HPV research in Slovenia," Acta Dermatovenerol Alp Panonica Adriat, vol. 20, pp. 99-112, 2011.

[3] N. Muñoz, F. X. Bosch, S. De Sanjosé et al., "Epidemiologic classification of human papillomavirus types associated with cervical cancer," The New England Journal of Medicine, vol. 348, no. 6, pp. 518-527, 2003.

[4] N. Muñoz, F. Méndez, H. Posso et al., "Incidence, duration, and determinants of cervical human papillomavirus infection in a cohort of Colombian women with normal cytological results," Journal of Infectious Diseases, vol. 190, no. 12, pp. 2077-2087, 2004.

[5] A. Ermel, B. Qadadri, A. Morishita et al., "Human papillomavirus detection and typing in thin prep cervical cytologic specimens comparing the Digene Hybrid Capture II Assay, the Roche Linear Array HPV Genotyping Assay, and the Kurabo GeneSquare Microarray Assay," Journal of Virological Methods, vol. 169, no. 1, pp. 154-161, 2010.

[6] C. Clavel, M. Masure, J. P. Bory et al., "Human papillomavirus testing in primary screening for the detection of high-grade cervical lesions: a study of 7932 women," British Journal of Cancer, vol. 84, no. 12, pp. 1616-1623, 2001.

[7] P. E. Castle, A. T. Lorincz, I. Mielzynska-Lohnas et al., "Results of human papillomavirus DNA testing with the Hybrid Capture 2 assay are reproducible," Journal of Clinical Microbiology, vol. 40, no. 3, pp. 1088-1090, 2002.

[8] A. Sargent, A. Bailey, A. Turner et al., "Optimal threshold for a positive hybrid capture 2 test for detection of human papillomavirus: data from the ARTISTIC trial," Journal of Clinical Microbiology, vol. 48, no. 2, pp. 554-558, 2010.

[9] F. Pei, X. P. Chen, Y. Zhang et al., "Human papillomavirus infection in nasal polyps in a Chinese population," Journal of General Virology, vol. 92, no. 8, pp. 1795-1799, 2011.

[10] K. Shigehara, T. Sasagawa, J. Doorbar et al., "Etiological role of human papillomavirus infection for inverted papilloma of the bladder," Journal of Medical Virology, vol. 83, no. 2, pp. 277285, 2011.

[11] K. Shigehara, T. Sasagawa, S. Kawaguchi et al., "Prevalence of human papillomavirus infection in the urinary tract of men with urethritis," International Journal of Urology, vol. 17, no. 6, pp. 563-568, 2010. 
[12] E. M. Ko, R. Tambouret, D. Wilbur, and A. Goodman, "HPV reflex testing in menopausal women," Pathology Research International, vol. 2011, Article ID 181870, 4 pages, 2011.

[13] O. Nunobiki, M. Ueda, E. Toji et al., "Genetic polymorphism of cancer susceptibility genes and HPV infection in cervical carcinogenesis," Pathology Research International, vol. 2011, Article ID 364069, 8 pages, 2011.

[14] H. C. Kitchener, M. Almonte, C. Thomson et al., "HPV testing in combination with liquid-based cytology in primary cervical screening (ARTISTIC): a randomised controlled trial," The Lancet Oncology, vol. 10, no. 7, pp. 672-682, 2009.

[15] N. Masumoto, T. Fujii, M. Ishikawa et al., "Dominant human papillomavirus 16 infection in cervical neoplasia in young Japanese women; study of 881 outpatients," Gynecologic Oncology, vol. 94, no. 2, pp. 509-514, 2004.

[16] G. A. Stanczuk, P. Kay, E. Sibanda et al., "Typing of human papillomavirus in Zimbabwean patients with invasive cancer of the uterine cervix," Acta Obstetricia et Gynecologica Scandinavica, vol. 82, no. 8, pp. 762-766, 2003.

[17] M. Q. Zhang, S. K. El-Mofty, and R. M. Dávila, "Detection of human papillomavirus-related squamous cell carcinoma cytologically and by in situ hybridization in fine-needle aspiration biopsies of cervical metastasis: a tool for identifying the site of an occult head and neck primary," Cancer, vol. 114, no. 2, pp. 118-123, 2008.

[18] R. M. Triglia, K. Metze, L. C. Zeferino, and L. A. L. Andrade, "HPV in situ hybridization signal patterns as a marker for cervical intraepithelial neoplasia progression," Gynecologic Oncology, vol. 112, no. 1, pp. 114-118, 2009.

[19] M. Poljak, I. J. Marin, K. Seme, and A. Vince, "Hybrid Capture II HPV Test detects at least 15 human papillomavirus genotypes not included in its current high-risk probe cocktail," Journal of Clinical Virology, vol. 25, Supplement 3, pp. S89S97, 2002.

[20] G. M. Clifford, J. S. Smith, M. Plummer, N. Muñoz, and S. Franceschi, "Human papillomavirus types in invasive cervical cancer worldwide: a meta-analysis," British Journal of Cancer, vol. 88, no. 1, pp. 63-69, 2003.

[21] K. W. K. Lo, Y. F. Wong, M. K. M. Chan et al., "Prevalence of human papillomavirus in cervical cancer: a multicenter study in China," International Journal of Cancer, vol. 100, no. 3, pp. 327-331, 2002. 

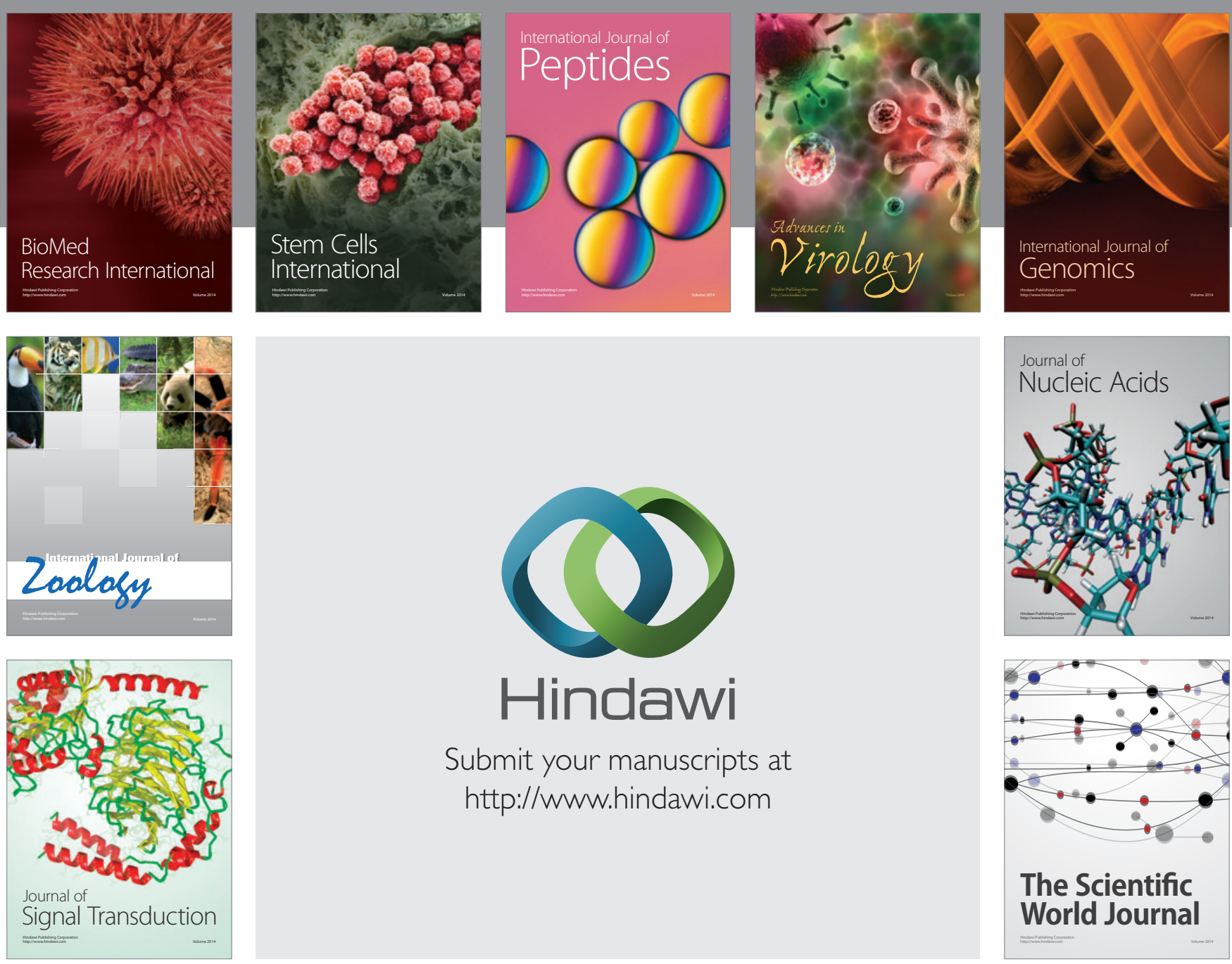

Submit your manuscripts at

http://www.hindawi.com
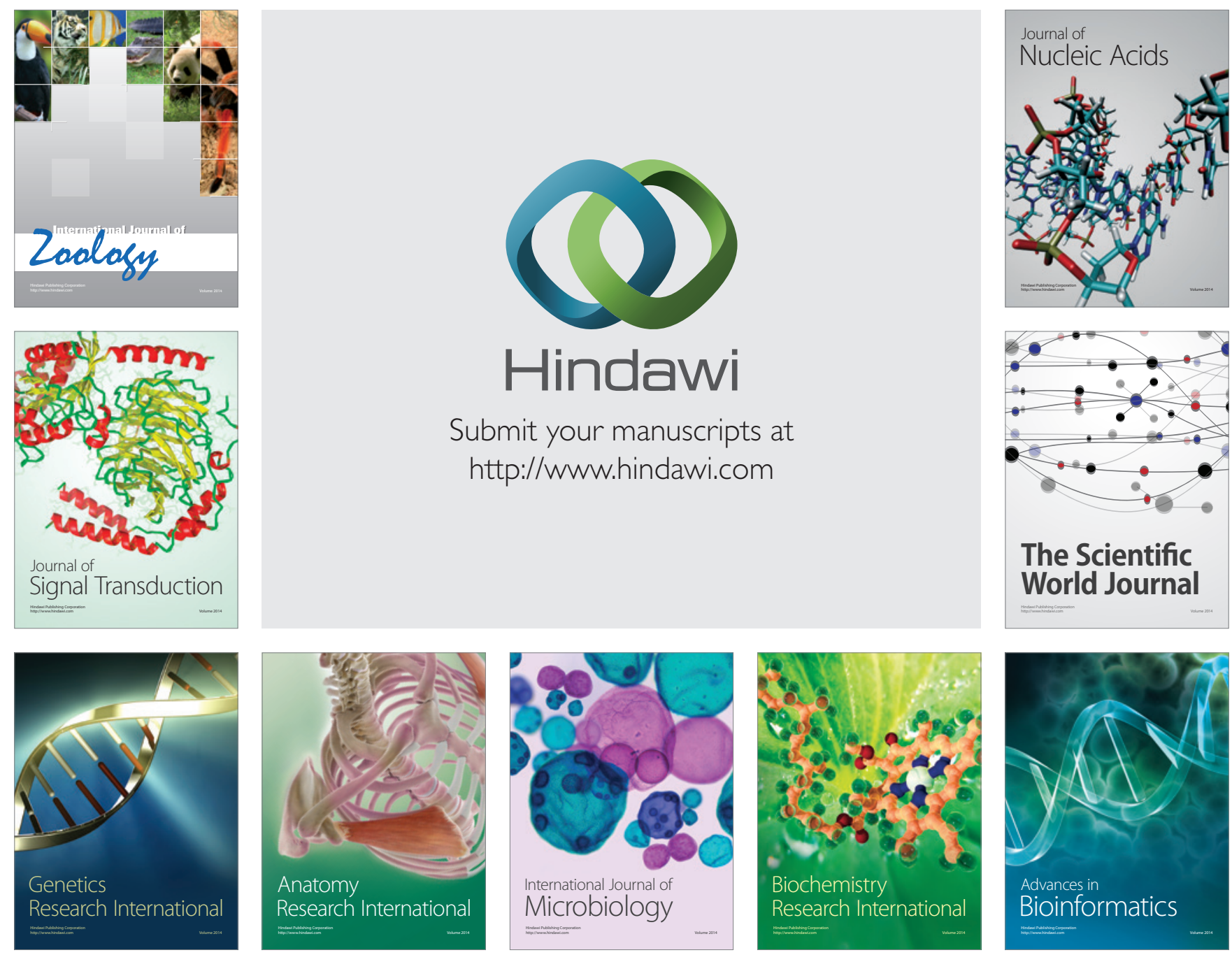

The Scientific World Journal
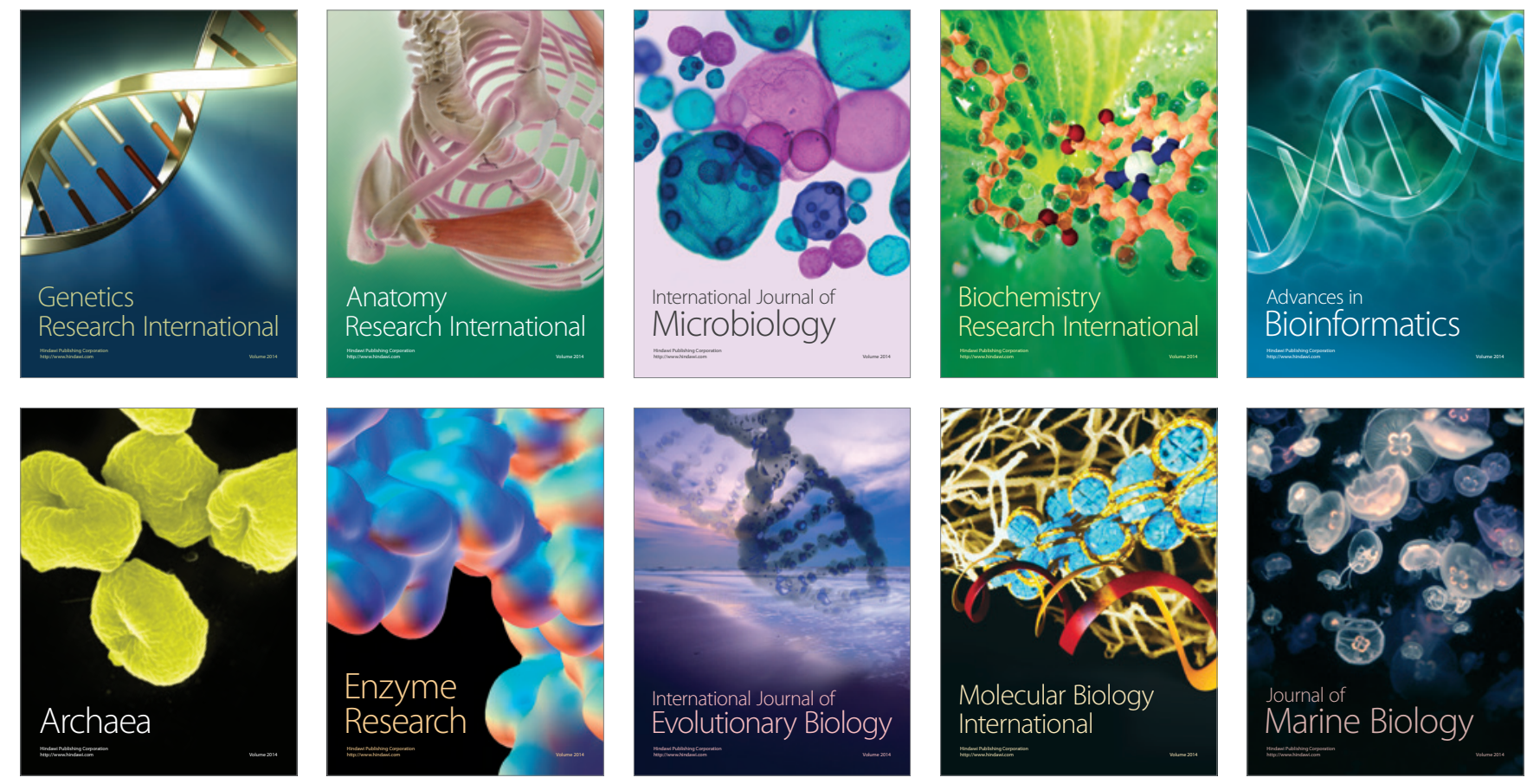